\title{
A REMARK ABOUT SEPARATION OF $K$-ANALYTIC SETS IN THE PRODUCT SPACES
}

\author{
LESZEK PIA̧TKIEWICZ
}

\begin{abstract}
A direct proof, using only "classical" notions, of a well-known theorem about approximation of coanalytic sets in the plane with "large" sections by Borel sets is given. A generalization of this theorem for hereditarily $K$-analytic spaces is also considered.
\end{abstract}

1. Introduction. It is an important and well-known theorem that if $C$ is a coanalytic set in the product of complete separable metric spaces whose vertical sections are "large" in the sense of category or measure then there exists a Borel set contained in $C$ whose vertical sections are "large" in the same sense (see Cenzer and Mauldin [2, Theorem 4.2], Dellacherie [3, Théorème 26, Appendice], Kechris [4]).

The proofs of that theorem we know split into two steps: the first step is a general result about an approximation of coanalytic sets in the products by Borel sets whose sections have a given property (see §3), and the second step is to demonstrate that if $A$ is an analytic set in the product $X \times Y$ of complete separable metric spaces, then the set of those points $x$ at which the section $A(x)$ is "large" in the sense of category (respectively, measure) is analytic in $X$, thereby to apply the result from the preceding step.

While there are proofs of the second step using only "classical" methods (e.g. a proof for the case of category due to Vaught [8, Theorem 1.6] and proof based on some simple reasoning related to capacity in the case of measure; cf. Bertsekas and Shreve [1, 7.43]); the published proofs of the first step we are aware of are based essentially on some "nonclassical" notions (see [2-4]).

In this note we present an argument which allows one to derive the conclusion needed in the first step directly from some classical facts related to the Lusin sieves.

Our reasoning can be carried out in a natural way for Hausdorff spaces whose open subsets are all $K$-analytic, which slightly extends the known result to a wider class of spaces.

I would like to thank R. Pol for some valuable discussions on the subject of this paper.

Received by the editors January 9, 1984.

1980 Mathematics Subject Classification. Primary 54H05; Secondary 03E15, 04A15, 28A05.

Key words and phrases. $K$-analytic sets, Lusin sieve. 


\section{Lusin sieves and $K$-analytic sets.}

2.1. Let $Q$ be the set of rational numbers. Given a space $X$ we shall call a collection $\mathbf{L}=\left\{L_{q}: q \in Q\right\}$ of closed subsets of $X$ a closed Lusin sieve in $X$ (cf. [5, $\S 3, \mathrm{XV} ; 7, \S 7])$.

Given a sieve $\mathbf{L}$, we let $\mathbf{L}(x)=\left\{q \in Q: x \in L_{q}\right\}$ for $x \in X$, and we define the index $I_{\mathbf{L}}(x)$ of $x$ with respect to $\mathbf{L}$ to be the order type of $\mathbf{L}(x)$ if this set is well ordered and $I_{\mathbf{L}}(x)=\omega_{1}$, the first uncountable ordinal, in the opposite case.

The set sifted by the sieve $\mathbf{L}$ in the space $X$ is the set $\mathbf{S}(\mathbf{L})$ of those points $x$ whose $\operatorname{index} I_{\mathbf{L}}(x)=\omega_{1}$.

Given a sieve $\mathbf{L}$ in the space $X$ we let $x \leqslant_{\mathbf{L}} y$ for $x, y \in X$ iff $I_{\mathbf{L}}(x) \leqslant I_{\mathbf{L}}(y)$, and let $\mathbf{G}\left(\leqslant_{\mathbf{L}}\right)$ be the graph of the relation $\leqslant_{\mathbf{L}}$, i.e. $\mathbf{G}\left(\leqslant_{\mathbf{L}}\right)=\left\{(x, y) \in X^{2}: x \leqslant_{\mathbf{L}} y\right\}$, we shall refer to $\leqslant_{\mathrm{L}}$ as to the partial order related to the sieve $\mathbf{L}$.

2.2. A set $A$ in a Hausdorff space $X$ is $K$-analytic if there exists an upper semicontinuous map $F: \omega^{\omega} \rightarrow \mathbf{K}(X)$ from the space of irrationals to the compact sets of $X$. such that $\bigcup_{t} F(t)=A$; for the basic properties of the class of $K$-analytic sets we refer the reader to a paper by Rogers and Jayne [6]. Let us recall that in the class of metrizable spaces $K$-analytic sets coincide with analytic sets, i.e. continuous images of the irrationals $\omega^{\omega}$.

2.3. If $A$ is a $K$-analytic set in a Hausdorff space $X$, then there exists a closed Lusin sieve $\mathbf{L}$ in $X$ such that $A=\mathbf{S}(\mathbf{L})$ is the set sifted by the sieve $\mathbf{L}$ (see [6, Theorem 2.5.2]).

2.4. If $\mathbf{L}$ is a closed Lusin sieve in a Hausdorff space $X$ such that each open set in $X$ is $K$-analytic, then the graph $\mathbf{G}\left(\leqslant_{\mathbf{L}}\right)$ of the partial order $\leqslant_{\mathbf{L}}$ associated with $\mathbf{L}$ is $K$-analytic. Indeed the assumptions allow one to repeat in this case a proof of the corresponding classical fact for analytic sets (see [5, §38, IX, Lemma 2]).

3. A separation result for $K$-analytic sets in the product spaces. Given a set $A \subset E \times F$ we shall write $A(x)=\{y \in F:(x, y) \in A\}$ for each $x \in E$.

3.1. TheOREM. Let $Z=X \times Y$ be the product of Hausdorff spaces such that each open set in $Z$ is $K$-analytic and let, for each $x \in X$, a collection $\mathbf{C}_{x}$ of subsets of $Y$ be given such that the following condition holds:

$$
\left\{\begin{array}{l}
\text { if } T \subset Z \times Z \text { is a } K \text {-analytic set, then the set } \\
T^{*}=\left\{(z, x) \in Z \times X: T(z, x) \notin \mathbf{C}_{x}\right\} \text { is } K \text {-analytic. }
\end{array}\right.
$$

Then, given a pair of disjoint $K$-analytic sets $A_{1}, A_{2} \subset Z$ such that $A_{1}(x) \in \mathbf{C}_{x}$ for each $x \in X$, there exists a Borel set $B \subset Z$ containing $A_{1}$ and disjoint from $A_{2}$ such that $B(x) \in \mathbf{C}_{x}$ for each $x \in X$.

3.2. Remark. The condition (+) in Theorem 3.1 is closely related to "definability properties" of Cenzer and Mauldin [2, Theorem 4.1] and to "modest collections" of Dellacherie [3, §43, Lemme 35] (see also §4), and the assertion of the theorem, at least for metrizable $Z$, can be achieved by the methods of those papers. Our main objective, however, is, as we indicated in the introduction, to give a direct proof of that fact. 
3.3. Remark. If $X$ is a Hausdorff space such that each open set in $X$ is $K$-analytic and $Y$ is a Hausdorff continuous image of the space of irrationals, then each open set in the product $X \times Y$ is $K$-analytic.

Proof OF Theorem 3.1. Let $\mathbf{L}$ be a closed Lusin sieve in the space $Z$ such that $A_{1}$ is the set sifted by $\mathbf{L}$, and let $\mathbf{G}\left(\leqslant_{\mathbf{L}}\right)$ be the graph of the partial order $\leqslant_{\mathbf{L}}$ associated with the sieve $\mathbf{L}$ (see §2.1).

Since each open set in $Z$ is $K$-analytic, the set $\mathbf{G}\left(\leqslant_{\mathrm{L}}\right)$ is $K$-analytic in $Z \times Z$ (see $\S 2.4)$, and the property $(+)$ guarantees that the set

$$
E=\left\{(z, x) \in Z \times X: \mathbf{G}\left(\leqslant_{\mathbf{L}}\right)(z, x) \notin \mathbf{C}_{x}\right\}
$$

is $K$-analytic and hence its projection on $Z$,

$$
A_{3}=\left\{z \in Z: \mathbf{G}\left(\leqslant_{\mathbf{L}}\right)(z, x) \notin \mathbf{C}_{x} \text { for some } x \in X\right\},
$$

is $K$-analytic as well.

Since, by the assumption, we have $\mathbf{G}\left(\leqslant_{\mathbf{L}}\right)(z, x)=A_{1}(x) \in \mathbf{C}_{x}$ for every $z \in A_{1}$ the sets $A_{1}$ and $A_{3}$ are disjoint, i.e.

$$
A=A_{2} \cup A_{3} \subset Z \backslash A_{1}=\left\{z \in Z: I_{\mathbf{L}}(z)<\omega_{1}\right\} .
$$

Now, let us put

$$
H=\left\{z \in Z: z \leqslant_{\mathrm{L}} z^{\prime} \text { for some } z^{\prime} \in A\right\} .
$$

Clearly, $H \subset\left\{z \in Z: I_{\mathbf{L}}(z)<\omega_{1}\right\}$ is a $K$-analytic set and, since $z \leqslant_{\mathbf{L}} z^{\prime}$ and $z^{\prime} \in H$ implies $z \in H$, there exists $z_{0} \in Z$ such that $H=\left\{z \in Z: I_{\mathbf{L}}(z)<I_{\mathbf{L}}\left(z_{0}\right)\right\}$. Let us consider

$$
B=Z \backslash H=\left\{z \in Z: z_{0} \leqslant_{\mathbf{L}} z\right\}=\mathbf{G}\left(\leqslant_{\mathbf{L}}\right)\left(z_{0}\right) .
$$

Since $\mathbf{G}\left(\leqslant_{\mathbf{L}}\right)$ is a $K$-analytic set, $B$ is $K$-analytic and, being the complement of $H$, it is Borel by a counterpart to the classical separation theorem due to Frolik (see [6, Theorem 3.3.1]).

Since $z_{0} \notin A_{3}, B(x)=\mathbf{G}\left(\leqslant_{\mathrm{L}}\right)\left(z_{0}, x\right) \in \mathbf{C}_{x}$ for each $x \in X$. Moreover, $H$ is disjoint from $A_{1}$ and it contains $A_{2}$, hence $B$ contains $A_{1}$ and is disjoint from $A_{2}$, so $B$ is the Borel set we were looking for.

4. Some applications. In this section we indicate, following Cenzer and Mauldin [2] and Dellacherie [3], some situations in which the separation result demonstrated in $\$ 3$ can be applied.

Throughout this section, $X$ is a Hausdorff space such that each open subset of $X$ is $K$-analytic and $Y$ is a metrizable $K$-analytic, i.e. analytic, space (cf. Remark 3.3).

4.1. The "modest collections" of Dellacherie. Let $\mathbf{F}(Y)$ be the family of all closed subsets of $Y$ endowed with the Effros Borel structure, i.e. with the $\sigma$-algebra generated by the sets $\{F \in \mathbf{F}(Y): F \cap U \neq \varnothing\}, U \subset Y$ being open.

Following Dellacherie [3, §43], let us call a family $\mathbf{C}$ of subsets of $Y$ a modest collection if

(i) given $E \in \mathbf{C}$ and $F \subset E, F \in \mathbf{C}$,

(ii) the intersection $\mathbf{C} \cap \mathbf{F}(Y)$ is a coanalytic set with respect to Effros Borel structure.

Given a modest collection $\mathbf{C}$ of subsets of $Y$, a standard verification shows that the collection $\mathbf{C}_{x}=\{E: \bar{E} \in \mathbf{C}\}$ for $x \in X$, satisfies the condition (+) in Theorem 3.1. This yields a variant of a separation theorem due to Dellacherie [3, §37]. 
Recall that examples of modest collections are in particular the class of relatively compact sets in the Lusin space, and the class of sets with empty interior (see [3, §43]).

4.2. The case of category. Let us assume in addition that $Y$ is a complete separable metric space. Then Vaught's formula [8, Theorem 1.6] easily yields that both collections

$$
\mathrm{C}_{x}=\{E \subset Y: E \text { is meagre in } Y\}, \text { for } x \in X,
$$

and

$$
\mathbf{C}_{x}^{\prime}=\{E \subset Y: E \text { is noncomeagre in } Y\}, \text { for } x \in X,
$$

satisfies the condition (+) in Theorem 3.1. As a result, we get a variant of an approximation theorem for coanalytic sets with sections "large" in the sense of category (see Cenzer and Mauldin [2, Theorem 4.2]).

4.3. The case of measure. Let $\mu: X \times \operatorname{Borel}(Y) \rightarrow R$ be a Borel measure distribution, (Borel $(Y)$ being the algebra of Borel sets in $Y$ ), i.e.

(i) $\mu_{x}=\mu(x, \cdot)$ : $\operatorname{Borel}(Y) \rightarrow R$ is a countable additive Borel measure for each $x \in X$,

(ii) $\mu_{B}=\mu(\cdot, B): X \rightarrow R$ is Borel measurable for any fixed Borel set $B$ in $Y$ (see $[2, \S 4])$.

Then, for each real number $r$, the collection $\mathbf{C}_{x}^{r}=\left\{E \subset Y: E\right.$ is $\mu_{x}$-measurable and $\left.\mu_{x}(E) \leqslant r\right\}$, for $x \in X$, satisfies the condition (+) in Theorem 3.1 (cf. Cenzer and Mauldin [2, Theorem 4.1] and Dellacherie [3, Théorème 24, Appendice]).

This fact can be verified by a direct reasoning related to the notion of capacity (see Bertsekas and Shreve [1, 7.43, 7.25]); more precisely one can modify in a straightforward way the reasoning presented there to extend the required result for the $K$-analytic case.

As a result, we get a variant of an approximation theorem for coanalytic sets with sections "large" in the sense of measure (see Cenzer and Mauldin [2, Theorem 4.2] and Dellacherie [3, Théorème 26, Appendice]).

\section{REFERENCES}

1. D. P. Bertsekas and S. E. Shreve, Stochastic optimal control. The discrete time case, Academic Press, New York, 1978.

2. D. Cenzer and R. D. Mauldin, Inductive definability: measure and category, Adv. Math. 38 (1980), 55-90.

3. C. Dellacherie, Un cours sur les ensambles analytiques, Analytic Sets (C. A. Rogers et al., eds.), Academic Press, London, 1980.

4. A. S. Kechris, Measure and category in effective descriptive set theory, Ann. Math. Logic 5 (1973), 337-384.

5. K. Kuratowski, Topology. I, PWN, Warsaw, 1966.

6. C. A. Rogers and J. E. Jayne, K-analytic sets, Analytic Sets (C. A. Rogers et al., eds.), Academic Press, London, 1980.

7. C. A. Rogers and R. C. Willmott, On the uniformizations of sets in topological spaces, Acta Math. 120 (1968), 1-52.

8. R. L. Vaught, Invariant sets in topology and logic, Fund. Math. 82 (1974), 269-294.

Institute of Mathematics, University of WaRSaw, Bialystok Branch, Ul. AKademicka 2 , 15 - 267 BialystoK, POLAND 